additional hazard after the disease has been present for ten years.

It is a radical concept which would connect the personality disorder with the malignant change, but such speculation, though idle, is of interest.

\section{K. GURLING.}

\section{REFERENCES} I. RICE-OXLEY, J. M., and TRUELOVE, S. (1950), Lancet,
i, 663 .

2. ANDRESEN, A. F. R. (1942), Am. ₹. Digest. Dis., 9, 91.

3. MURRAY, C. D. (1930), F. Nerv. Ment. Dis., 72, 617.

4. MURRAY, C. D. (1930), Am. F. Med. Sci., 180, 239.

5. SUllivan, A. J. (1936), Am. F. Digest. Dis., 2, 65 I.

6. DANIELS, G. E. (1942), New England F. Med., 226, 178.
7. WITTKOWER, E. (1938), Brit. Med. F., ii, 1356.

8. GRACE, W. J., WOLF, S., and WOLFF, H. G. (I951), ' The Human Colon,' Heinneman, London.

9. LIUM, R. (1939), Am. F. Med. Sci., 197, 841.

ro. LIUM, R. (1939), Arch. Int. Med., 63, 210.

II. FLEMING, A. B. (1922), Proc. Royal Soc., S.B., 306.

12. MEYER, K., and HAHNEL, E. (1946), f. biol. Chem., 163, 723.

13. SAMMONS, H. G. (195 I), Lancet, ii, 239.

14. PAULLEY, J. W. (1950), Gastroenterology, 16, 566.

r 5. HINTON, J. W., and SHARIHOFF, B. P., cited by Grace, Wolf and Wolff (8).

I6. MORTON-GILL, A. (1944), Lancet, i, 536.

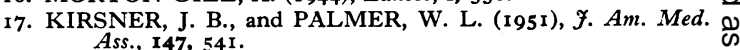

18. HARDY, T. L., and BROOKE, B. (1952), 'Modern Trends in Gastroenterology,' edited by F. Avery Jones; Butterworth, London.

19. LAHEY, F. H. (1950), Rev. Gastroenterology, 17, 723.

20. SLOAN, W. P., BARGEN, J. A., and CAGE, R. P., (1950) Gastroenterology, 16, 25.

\title{
THE TREATMENT OF LUMBAR DISC-LESIONS
}

By James Cyriax, M.D.

Physician to the Department of Physical Medicine, St. Thomas's Hospital

The discovery that the common cause of backache and sciatica is a disc-lesion has caught the medical profession largely unprepared. For centuries these symptoms had been considered a reason for instituting treatment by heat, massage and exercises. These methods have now lost their last shred of theoretical justification but are being replaced only very slowly by acceptable alternatives. Nevertheless, a number of simple measures exist, none a panacea, each with its due proportion of successes. Few patients cannot be relieved if conservative treatment is intelligently used, and it is for only a few of this remainder that surgery need be considered.

\section{Prophylaxis}

The direction in which a damaged disc tends to. move depends on the forces acting on it. During lordosis the inclination of the joint surfaces (Fig. I) is such that the pressure of the body weight on the joint pushes the disc anteriorly. During kyphosis (Fig. 2) the tilt becomes reversed and the disc is pushed backwards. Hence it is only postures involving flexion that are apt to result in posterior displacement. The dura mater and the nerve roots lie posteriorly and are the sensitive structures that make the patient aware of disc-protrusion, the lumbar joints being in themselves all but insensitive. The important movement to avoid is stooping, especially when weight-lifting increases the compression force or the joint. Industrial medical officers should tea workers how to lift, using their knees rather than their backs (Fig. 3), should see to it that heavy objects are presented at a suitable height and

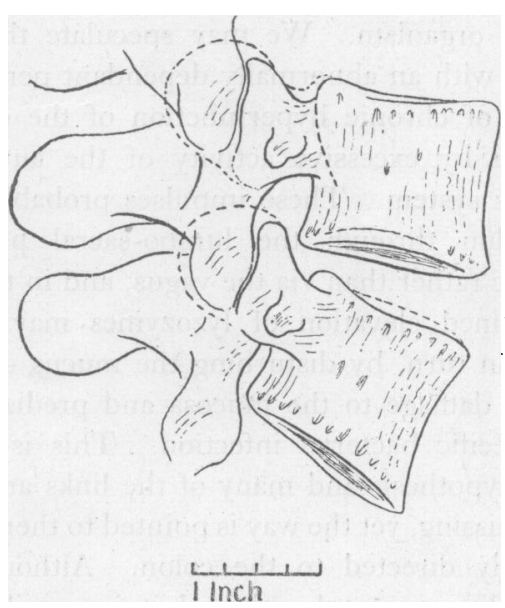

FIG. I.- Joint space in extension. Tracing of X-ray photograph of the fourth lumbar intervertebral $\Phi$ joint in a normal subject, bending backwards. $\stackrel{\text { ? }}{+}$ Note that the front of the joint space is $\frac{1}{2}$ in. 0 $(1.25 \mathrm{~cm}$.) wide, but the back only $3 / 16$ in. $(0.5 \mathrm{~cm}$.). In this position backward movement of the intervertebral disc is virtually impossible. 


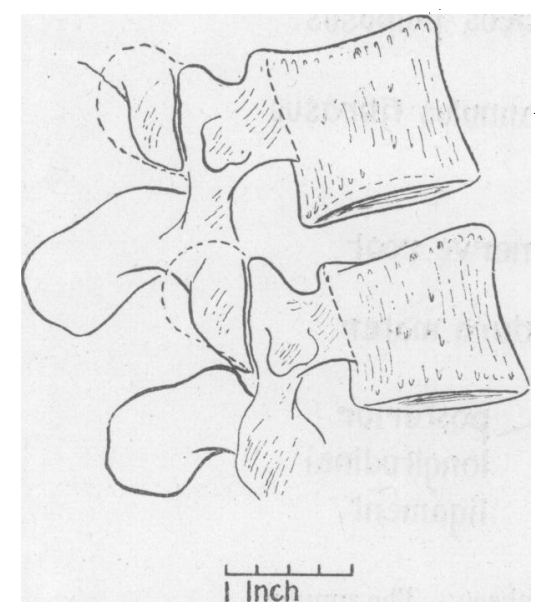

Fig. 2.- Joint space in flexion. The same subject as in Fig. I bending forwards. The joint space is now $5 / 16$ in. $\left(0.8 \mathrm{~cm}\right.$.) wide behind, and $\frac{1}{4}$ in. $(0.6 \mathrm{~cm}$.$) in front.$

attend to the design of chairs and car seats. Patients in bed should not be allowed to lie in 'the nursing mother's position,' i.e. half-sitting with many pillows behind the thorax and no support for the lumbar spine which droops into kyphosis all day. No wonder the posterior longitudinal ligament finally stretches and the beginnings of disc-protrusion are laid down. The lithotomy position and the flexion that some patients are made to maintain during lumbar puncture are obvious offenders. Nurses require instruction on these points and it should be explained to gymnasts giving children postural training at school that the lordosis is not an unsightly curve requiring obliteration but the chief mechanical bar to disc-protrusion.

\section{Treatment}

If something is out of place the most obvious approach is to put it back again. This concept governs the orthodox immediate treatment of most fractures and of rupture with subluxation of the meniscus at the knee. When it comes to the spinal joints an extraordinary hiatus exists. Even after a doctor has arrived at a correct diagnosis he regards active measures to promote reduction as so unorthodox that he does not attempt them at all. This remarkable attitude has brought into being a group of laymen-variously called bonesetters, osteopaths or chiropractors - who carry out to our recurrent discomfiture the simple spinal manipulations that we in general avoid. Lately we have explained away our neglect by dilating on the dangers of manipulating the spinal joints-to the great advantage of those laymen who, without

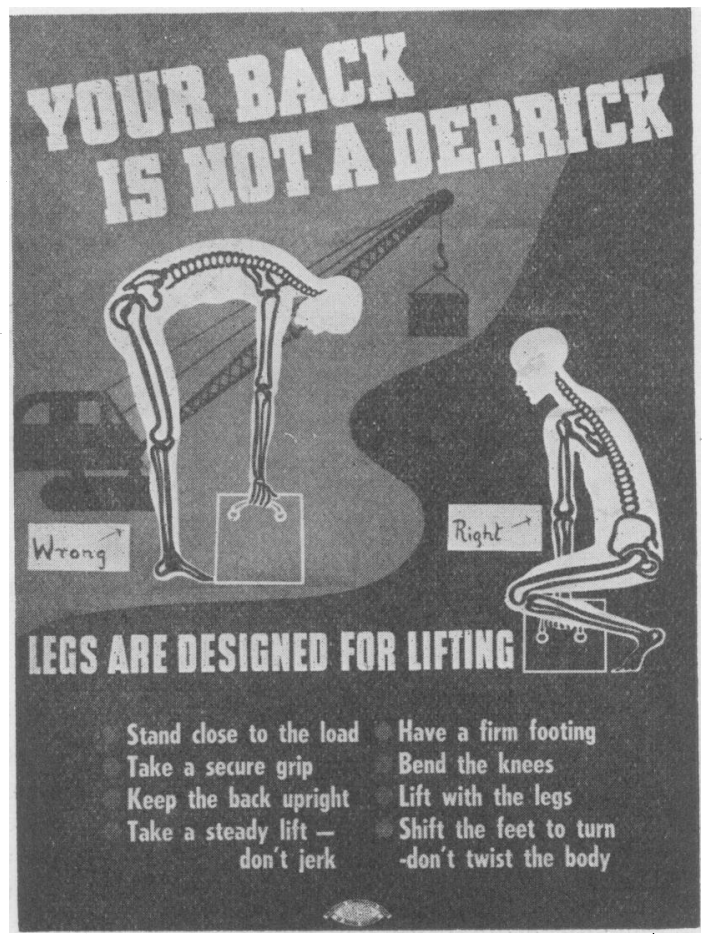

FIG. 3.-A Canadian poster.

realizing it, have for years been reducing minor subluxations of part of the intervertebral annulus.

The first decision that has to be arrived at is whether the displacement is likely or unlikely to be reducible by manipulation. The reason for the two different responses to manipulation is anatomical. Displacements of the annulus (Fig. 4) consist of hard material that moves under the stresses of manipulation; protrusions of the nucleus usually do not for they have the consistency of wet sand (Fig. 5). Burns and Young (1945), analyzing cases coming to laminectomy, found that the primary lesion in 56 per cent. of their cases to be cartilaginous, and in 44 per cent. to be pulpy with an intact annulus. My impression is that in patients not requiring laminectomy the proportion is more like one nuclear protrusion to two annular. Thus the first major decisionwhether or not manipulation should be attempted -rests on an evaluation of the symptoms and signs differentiating a nuclear from an annular protrusion. These are set out overleaf.

\section{Differentiation of Cartilaginous from Pulpy} Protrusions

History

This is often indicative. For example, a patient 


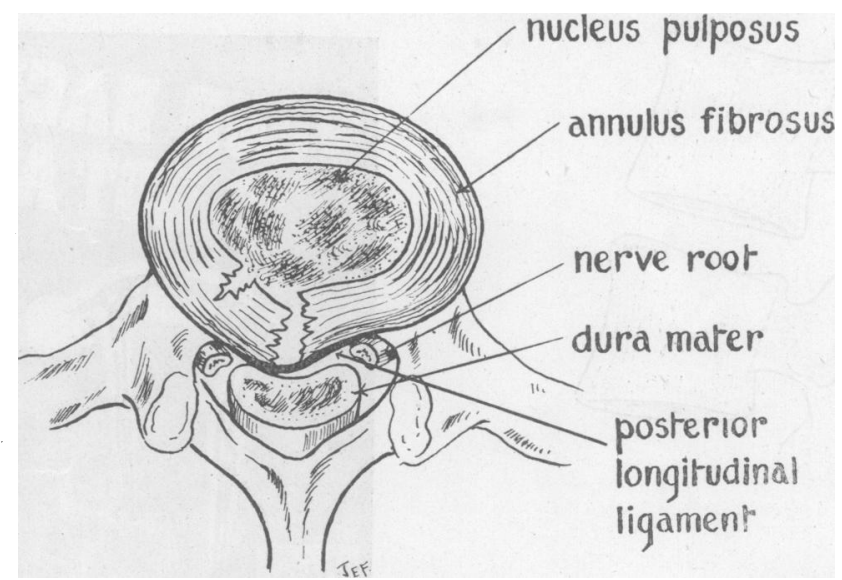

Fig. 4.-Cartilaginous protrusion causing lumbago. The annulus is cracked and hinged. Reduction by manipulation should be simple.

bends forwards and feels some aching in his back which gets worse later in the day. Next morning he finds himself unable to get out of bed because of severe lumbago. This history indicates a protrusion that has gradually increased in size - that is; one consisting of nuclear material. By contrast, the patient who is subject to attacks initiated by a click in the back follawe by agonizing lumbar pain fixing him in flexion has clearly suffered an abrupt cartilaginous displacement. Pulpy protrusions are uncommon in the elderly, hence manipulative reduction should always be attempted for displacements, whether causing backache, gluteal pain or sciatica in patients over 60 years old.

Primary postero-lateral protrusions causing sciatica are irreducible by manipulation. This is indicated when a patient with a low lumbar disclesion states that his pain began in the calf or thigh and does not reach the back. Naturally a central displacement impinges first against the dura mater, causing backache before it sets up sciatica; primary postero-lateral protrusions never touch the dura at all, hence premonitory backache is absent.

Another type of history characterizes the selfreducing pulpy disc-lesion; in such a case manipulation is waste of time, achieving no more than the patient achieves for himself by avoiding compression. The patient wakes comfortable and as the day goes on backache appears and becomes slowly more severe, especially if he stoops or lifts. A night's rest once more abolishes the pain. Naturally, if the posterior bulge at the joint recedes spontaneously as soon as the stress on the joint ends, only to recur when the joint is made to bear weight again, the reduction brought about by manipulation is equally unstable and ephemeral.
Manipulation carried out at a time when no displacement is present is, of course, quite pointlesse

A similar history characterizes what I have named the 'mushroom phenomenon' (Cyriax, I950). In an elderly patient, backache soon followed by bilateral sciatica comes on after aboutô minutes' walking or standing and is abolished $a \mathrm{~s}^{\prime}$ soon as he rests. It is often mistaken for interi mittent claudication. X-ray examination show that the joint space at, usually, the fifth lumba level has disappeared, the disc substance being displaced forwards and lying as a round ball be $\bar{\alpha}$ tween two large osteophytes. Since nothing sensitive is touched, anterior protrusion proceeds silently, causing no symptoms until the disc hasbeen ground to pieces and the compression of weight-bearing causes capsular bulging all the way round the joint. The posterior component then. irritates both the dura mater and the nerve rootsiThis lesion does not respond to manipulation.

A complaint of weakness of the bladder, saddle anaesthesia, paraesthesiae in the scrotum or numb ness of the labium shows that the fourth sacrab root is squeezed and provides an absolute contra? indication to manipulation. The significance of referred rectal, penile, or coccygeal pain is less clear, but may also indicate a protrusion im perilling the fourth sacral root. Since the important danger of manipulation in an unsuitable case is lasting urinary incontinence, I regard this ${ }^{\omega}$ type of referred pain as contraindicating manipulation. Not having attempted it in such a case naturally cannot be sure to what extent this? apparent danger is real.

Signs

Of the physical signs the appearance of the 


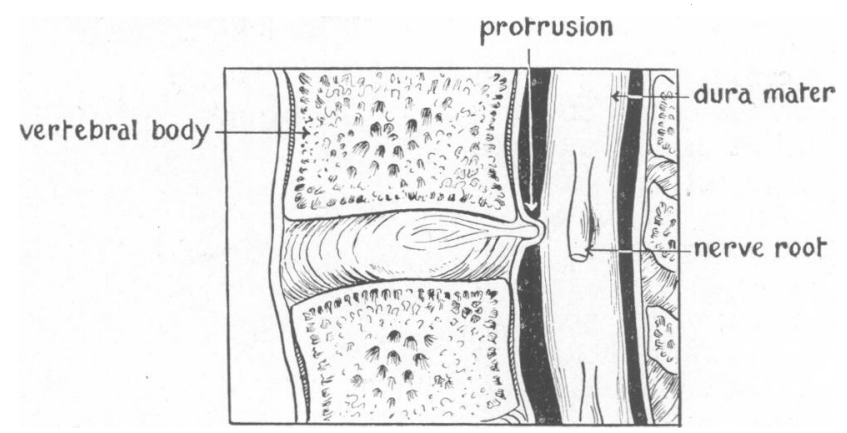

Fig. 5.-Pulpy protrusion causing lumbago. The nucleus has protruded backwards, the annulus remaining intact (after R. H. Young). Manipulation is unlikely to succeed; sustained traction or recumbency is effective.

patient's back is the most informative. Lateral deformity of the lumbar spine when he is viewed standing still, or apparent when he bends forwards, indicates a protrusion that has reached a size that visibly interferes with joint movement. Considerable lateral deviation therefore indicates irreducibility. I have also found that reduction by manipulation is difficult, at times impossible, in patients whose lumbar movements (other than flexion) hurt in the thigh instead of the back or in whom the most painful movement is sideflexion towards the painful side.

Marked neurological signs should be taken as an indication of irreducibility, whether the lesion was originally of cartilage or pulp. Displacement of a fragment of annulus may later be followed by extrusion of nuclear material along the line of the fracture. This leads to severe compression of the nerve root and proportionate interference with conduction. Thus weakness of more than one muscle in the leg, combined with, say, loss of ankle jerk, cutaneous analgesia or much gluteal wasting shows that this secondary effect has supervened.

A disc-lesion at the unstable joint resulting from spondylolisthesis is treated in the same way as an ordinary disc-lesion.

\section{Manipulation}

This is the treatment of choice; for it can be immediately effective. It is carried out in all suitable cases as soon as the diagnosis of an annular displacement is made.

\section{General Anaesthesia}

This must be avoided, since it deprives the manipulator of the patient's co-operation, so vital to effective work. One manoeuvre is tried, whereupon the patient stands and by repeating the move- ments previously found painful assesses the resul on his symptoms; the manipulator watches the effect on his range of straight-leg raising, posture and lumbar mobility. If this manipulation has done good, it is repeated until no further benefit accrues. The next is then performed and the result noted again. In this way the manipulator sees what results he is achieving and knows what to do next, when to go on and when to stop. Anaesthesia denies him all this essential knowledge with the result that it is not difficult to make the patient, at least temporarily, worse. It is my experience, too, that the muscular relaxation induced by general anaesthesia is not of much advantage, for displacements previously reduced under anaesthesia have proved equally easy to reduce without, and failure without anaesthesia leads to failure with anaesthesia. It is not the manipulator or the patient's relaxation that is at fault but the type of protrusion. If it is large and pulpy no amount of manipulation has any chance of success.

\section{Technique}

The patient lies prone on a firm couch ${ }_{5}$ in. $(38 \mathrm{~cm}$.) high and various manual pressures are applied at the appropriate lumbar level. If these fail, rotation strains should be tried. These methods have been described and illustrated (Cyriax, 1950). This treatment may require two or three repetitions and an adequate session may last half an hour, hence it is my practice to delegate much of this work to physiotherapists trainedas all our students are-in these methods. Not all doctors have time, inclination or a suitable couch for carrying out such manoeuvres themselves; moreover these have in practice been found suited to delegation. 


\section{Sustained Traction}

By this means a patient can, as it were, be put to bed for several weeks in the course of a few hours. Most pulpy protrusions should be treated by immediate traction, carried out daily until reduction is complete. They ooze out and are by this means squeezed back into place again. Traction has two effects: (I) Distraction of the vertebral bodies (Fig. 6). (2) Tautening of the posterior common ligament which exerts contripetal force on the nuclear protrusion and slowly pushes it back. The increased distance apart of the vertebral bodies not only enlarges the space into which the protrusion can now return but creates suction.

The couch is made of steel and has a pillar with a hook on it at each end. One hook can be made to travel distally by rotating a wheel. For pulpy lumbar herniations a band is applied round the patient's lower thorax and fixed to the upper hook, another about his pelvis passes to the lower hook. Between 100 and $200 \mathrm{lb}$. traction is applied for half to one hour-as long as the patient can comfortably stand it. In an emergency up to six hours' continuous traction can be borne. The physiotherapist stays with the patient and adjusts the tension, measured on a spring balance, as required. This balance is important, not only to enable accurate treatments to be given but it takes up slack should the belt slip slightly and prevents all traction being temporarily lost.

\section{Contra-indications}

Cartilaginous displacements should be manipulated back into place. Elderly patients or those who have had thoracic operations may find that the band round the chest embarrasses respiration. Acute lumbago with twinges is unaffected or made worse. A patient with sciatica due to a pulpy protrusion of several months' standing is often in slight pain only, though the signs are marked. Traction may effect reduction, but a protrusion reduced may move again. By contrast if spontaneous cure is awaited, though it often takes a year from the onset of root pain, the tendency to recurrence is very slight. Hence there comes a time in the evolution of a pulpy protrusion when it is better policy to avoid even effective treatment for the sake of the long-term result.

\section{Epidural Local Anaesthesia}

The injection for epidural analgesia is regarded as difficult and dangerous by some authorities. During the last 12 years I have used this method on unprepared out-patients more than 10,000 times without ill effect, and have found it impossible to introduce the needle properly in less than I per cent. of all cases. The patient can walk

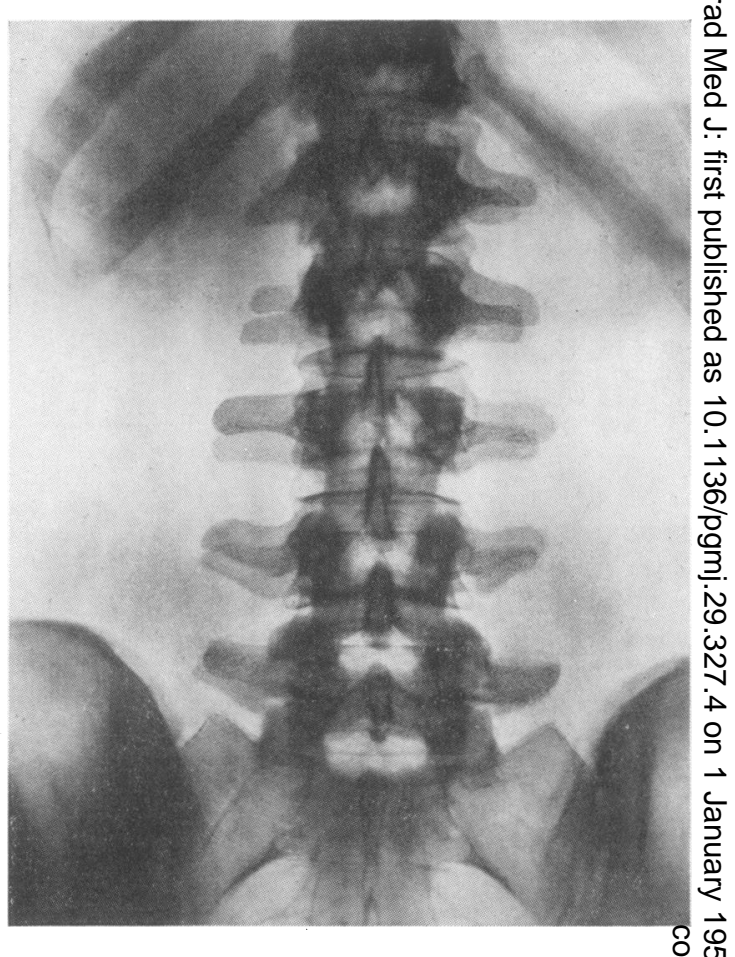

FIG. 6.-Two radiographs have been superimposed, sacra coinciding. One was taken before, the other during, traction.

home about half an hour after the injection is given. There are five main indications:

I. In acute lumbago the cause of the pain is a $\overrightarrow{\overrightarrow{0}}$ central posterior protrusion impinging on the dura 3 mater (Cyriax, 1945). If this membrane is anaesthetized by $50 \mathrm{ml}$. of 0.5 per cent. procaine, introduced extrathecally via the sacral canal, the $\frac{0}{3}$ protrusion presses on a membrane no longer: sensitive and all pain ceases for the duration of the 3 analgesia - that is, one to two hours. During this time a patient can move freely, perform some essential work, or go home to bed. He can with advantage lie on the couch for an hour in hyper-? extension, thus initiating reduction. If this is $\frac{7}{0}$ done he usually experiences a large measure of $=$. lasting relief.

2. Persistent lumbar or sciatic aching after reduction of a prolapsed disc may prove trouble- $N$ some; it is apparently due to persistent local $\omega$ bruising of the dura mater. One or two epidurale injections usually suffice to stop it.

3. Chronic backache, especially if it is more severe at night than by day, associated with only ${ }^{-}$ slight articular signs at the lumbar spine can oftenbe lastingly abolished by one injection of epidural local analgesia. 
4. If a patient in whom spontaneous recovery from sciatica is being awaited continues too long to suffer marked limitation of straight-leg raising, the nerve root can be painlessly mobilized by full straight-leg raising after the induction of an epidural block.

5. This is the treatment of choice in referred coccygodynia.

\section{Prolonged Analgesia}

This is indicated in large irreducible protrusions especially at the fourth lumbar level. The patient often suffers little pain but has a gross lateral deviation of the lumbar spine which may have persisted unaltered for weeks or months.

He lies supine in bed in his position of ease. $\mathrm{He}$ is kept fully relaxed for four hours by a continuous intravenous infusion of procaine solution. At the end of the third hour his position in bed is altered to the reverse of that obtaining previously, so that the lumbar spine is held in lateral deviation in the opposite direction. Prolonged muscular relaxation often results in reduction.

\section{Rest in Bed}

This is the old-fashioned treatment for lumbago and sciatica. It acts by avoiding the compression of standing; the vertebral bodies finally move apart and reduction ensues. It is a most successful treatment, but very slow. It is apt to fail only in the elderly whose lumbar joints are so stiff as the result of capsular contracture that they cannot move adequately. In such patients a fragment of annulus that weeks in bed have failed to shift can often be reduced easily by manipulation.

Rest in bed is an admission of defeat and often takes weeks or months to become effective. It is, therefore, to be avoided as far as possible. If there is nothing else for it, the patient must lie flat on a firm mattress, with a small pillow or a hot-water bottle maintaining his lordosis. He must not get out of bed nor sit up or the relief from compression at the joint is nullified. No sort of physiotherapy is called for and all exercises, even towards extension lying prone, are contra-indicated, for contraction of the sacrospinalis muscle squeezes the joints together and extends the joints only when this play has been taken up.

\section{Awaiting Spontaneous Cure}

Backache shows little tendency to spontaneous cure; it may last, or recur, during a patient's whole lifetime. Sciatica, however, nearly always gets well of itself in about 12 months. The two exceptions are: (a) after an unsuccesful laminectomy this does not happen and sciatica may then persist for years; and (b) after the age of 60 spontaneous cure with the lapse of time is improbable.

Young patients with sciatica are often seen who show clear signs of a nuclear protrusion at a lower lumbar level of some months' standing. The symptoms amount to only an ache, the patient looks cheerful and sleeps well. Examination standing shows a symmetrical lumbar spine with some lateral deviation on attempted flexion. Straight-leg raising is $45^{\circ}$ to $60^{\circ}$ limited and slightly impaired conduction is noted, e.g. a sluggish ankle-jerk and some weakness of the extensor hallucis muscle. As already mentioned, awaiting spontaneous recovery has a more permanent result than such treatments as bring about reduction, for the protrusion becomes fixed in a new position from which it cannot become dislodged.

The tendency to lumbago often ceases spontaneously between the ages of 50 and 6o. As age advances the spinal joints stiffen and osteophytes, both cupping the disc and limiting articular movement, make their welcome appearance. Osteophyte formation at the lumbar spine is not a painful condition, on the contrary it is beneficial. It is the mechanism that prevents backache after years of wear and tear have damaged the disc.

\section{Laminectomy}

This is to be avoided as far as possible, but operation should not be unreasonably withheld. In the best hands the results are not always perfect; immediate cure may not be secured and eventual recurrence is regrettably frequent even in patients who never go back to heavy work. The introduction of sustained traction in I950 reduced my laminectomy rate for all disc-lesions from $I: 40$ to $I: 200$. The five main indications are:

I. Gross lumbar deformity. A young patient should not be left to recover spontaneously from sciatica or, less often, backache causing marked persistent lumbar deformity. Though he may recover as regards pain, the deformity remains permanent.

2. Incipient drop foot. A patient who develops increasingly pronounced weakness of the dorsiflexor muscles of the foot must be warned so that he must choose between laminectomy and the possibility of a permanently weak foot. In such cases pressure atrophy, doubtless from local ischaemia, may result in complete insensitivity of the sheath of the affected nerve root. As a result, pain quickly ceases and straight-leg raising soon reaches full range at the same time as the palsy becomes complete. The patient, chiefly concerned with his pain, is apt mistakenly to suppose that he is getting better, an error that the physician shares 
unless he examines again the power of the muscles controlling the foot.

3. Intractable severe pain. Irreducible disclesions are caused by complete erosion of the posterior ligament with extrusion of part of the annulus into the neural canal. More rarely the end-plate becomes detached and buckles over on itself. No amount of conservative treatment can then avail.

The important point in such cases is to make sure that the allegations of pain are borne out by the physical signs. Patients with hysteria welcome any operation the criterion of whose success rests almost entirely on the patient's own statements.

4. Fourth sacral palsy. Weakness of the bladder, saddle anaesthesia, paraesthesiae in scrotum or labium call for immediate laminectomy.

5. Adherent root. If the symptoms warrantthey usually do not-the adhesions can be divided at laminectomy.

\section{Arthrodesis}

Arthrodesis is the operation of choice in spondylolisthesis causing sufficient local capsular pain or setting up traction on the relevant lumbar nerve roots with consequent bilateral sciatica. It is the only effictive treatment for anterior protrusion of the whole disc (mushroom phenomenon), and is well worth carrying out in patients under 6o. It is also indicated in recurrence after laminectomy if conservative measures fail. Frequent crippling attacks of lumbago or sciatica quickly recovering present a difficult problem. Laminectomy rures less than half of all such cases. Arthrodisis is therefore to be preferred.

\section{Maintenance of Reduction \\ Explanation}

The mechanics of lumbar disc-protrusion must be explained to the patient so that he understands why it is essential that he should maintain his lordosis at all times. The physiotherapist shows him how to stand, sit, lift and how to use his knees instead of his lumbar joints for getting his hands close to the floor. Neurosis is prevented by emphasizing the purely mechanical nature of his disorder-the analogy with the knee is usefuland that it is not the precursor of arthritis likely to spread to other parts of the body. It is not so much that he cannot do this or that, he must do it in a different way. All exercises, especially towards trunk flexion, must be avoided. They maintain mobility and thus enable the movement to take place that results in internal derangement. Active prone-lying trunk extension exercises are $\frac{3}{8}$ less harmful than the others, but are best avoided $\stackrel{\varnothing}{\varnothing}$ since the contraction of the sacrospinalis muscles compresses the joint. Press-ups, where by the trunk is passively extended, are free from this defect.

\section{Support \\ A Plaster Facket}

This is a method in great vogue at the moment, but I believe it is seldom worth the discomfort it $\vec{\circ}$ entails. It is usually wrongly prescribed in the hope of achieving reduction rather than main- $\mathscr{O}$ taining it. One untenable reason for immobilization in plaster has been expressed (Crisp, 1948) that rest in plaster allows the broken cartilage to is unite. Intra-articular cartilage has no blood $\underset{\omega}{\oplus}$ supply; therefore it cannot heal at a spinal joint $\mathrm{N}$ any more than at the knee joint. Immobilization is achieves a spurious popularity when a patient, for 은 whom the proper treatment is to await spontaneous cure, is put in plaster until he is well. He does not know that he would have recovered just as quickly without the added discomfort of the plaster jacket.

\section{A Perforated Plastic Facket}

This weighs a fifth as much as a plaster jacke and achieves a much greater degree of immobiliz每: tion; for it can be tightened at will to any degree It can be made in two days on a plaster cast taken from the patient. Hence it supersedes a plaster jacket from every point of view.

\section{$A$ Corset}

Whereas a plaster jacket cannot be worn for longer than some months and thus cannot provide lasting protection against recurrence, corsets, renewed each year or two, can be worn indefinitely, thus affording permanent security. If the two steels are accurately moulded to the lumbar curve, 3 . lordosis is maintained and the joints steadied. Moreover, if the patient bends too far forwards or sags as he sits, the front of the corset presses unpleasantly against his lower ribs-a salutary reminder.

BIBLIOGRAPHY

BURNS, B. H., and YOUNG, R. H. (1945), 'Protrusion of Intra- O vertebral Disc,' Lancet, ii, 424.

CRISP, E. J. (I948), 'Conservative Treatment of Lumbar Disc Lesions,' Proc. Koy. Soc. Med., $26 \mathrm{I}$.

CYRIAX, J. (1945), ' Lumbago,' Lancet, ii, 426.

CYRIAX, J. (1950), ' Lumbar Disc Lesions,' Brit. med. F., ii, I434 CYRIAX, J. (1950), 'Orthopaedic Medicine,' Vol. II, Cassell, London. 